\title{
Targeted agents or immuno-oncology therapies as first-line therapy for BRAF-mutated metastatic melanoma: a real-world study
}

\author{
Jason J Luke*,1, Sameer R Ghate ${ }^{2}$, Jonathan Kish ${ }^{3}$, Choo Hyung Lee ${ }^{3}$, Lindsay McAllister ${ }^{3}$, \\ Sonam Mehta ${ }^{3}$, Briana Ndife ${ }^{2} \&$ Bruce A Feinberg $^{3}$ \\ ${ }^{1}$ Hematology \& Oncology (Cancer), University of Chicago Comprehensive Cancer Center, Chicago, IL 60637, USA \\ ${ }^{2}$ Novartis Pharmaceuticals Corporation, East Hanover, NJ 07936, USA \\ ${ }^{3}$ Cardinal Health Specialty Solutions, Dublin, OH 43017, USA \\ *Author for correspondence: jluke@medicine.bsd.uchicago.edu
}

\begin{abstract}
Aim: Targeted therapy (TT) and immuno-oncology (IO) drugs are approved for patients with BRAF mutant metastatic melanoma (MM). We compared real-world outcomes for first-line (1L) TT versus $1 \mathrm{~L}$ IO to evaluate optimal sequencing. Materials \& methods: Physicians-identified BRAF mutant MM patients initiating $1 \mathrm{~L} \mathrm{TT}$ or 10 therapies and extracted treatment, disease and clinical outcomes including disease response which were compared between TT and 10 and individual regimens. Results: $440 \mathrm{MM}$ patients ( $\mathrm{TT}=283$, $I O=157)$ were identified. A higher proportion of TT patients had liver metastases ( 46.3 vs $35.0 \%)$ and abnormal lactate dehydrogenase (61.1 vs $42.7 \%$ ). IO-treated had a RECIST-determined response rate of 45.9 versus $60.1 \%$ for TT and time on treatment of 7.2 versus 11.4 months, respectively. There was no survival difference between cohorts. Conclusion: Despite higher risk patients, $1 \mathrm{~L}$ TT resulted in higher response rate and longer treatment duration suggesting a preferred $1 \mathrm{~L}$ sequence.
\end{abstract}

First draft submitted: 28 December 2018; Accepted for publication: 29 January 2019; Published online: 25 February 2019

Keywords: BRAF • cobimetinib • dabrafenib • ipilimumab • metastatic melanoma $\bullet$ nivolumab $\bullet$ pembrolizumab • trametinib • vemurafenib

In just 4 years, seven drugs received US FDA approval and labeling for the treatment of patients with BRAF mutant metastatic melanoma (MM) and within two years all had a first-line (1L) indication. Since the approval of the BRAF inhibitor dabrafenib (D) and the MEK inhibitor trametinib (T) the National Comprehensive Cancer Network (NCCN) has given a category 1 recommendation for 1L BRAF/MEK combination therapy [1] with $\mathrm{D}+\mathrm{T}[2,3]$ or vemurafenib plus cobimetinib $(\mathrm{V}+\mathrm{C})$ [4]. Additionally, the NCCN recommends immuno-oncology (IO) therapies including single-agent programmed cell death protein 1 (PD-1) treatment with nivolumab (nivo) [5], pembrolizumab (pembro) [6], or the combination of ipilimumab, a CTLA-4 inhibitor, plus nivo (ipi/nivo) [5]. In June 2018, an additional combination targeted therapy (TT), encorafenib plus binimetinib was approved for the treatment of BRAF mutant MM [7].

No head-to-head randomized controlled trials (RCTs) comparing TT and IO for BRAF mutant MM have been conducted. As such, there is no consensus on the optimal sequencing of $1 \mathrm{~L}$ and subsequent treatment for these patients, nor is it known how patients outside of the RCT setting have responded to these novel agents [8]. Treatment comparisons via prospective trials, such as the ongoing ECOG-ACRIN trial NCT02224781 (randomization to 1L $\mathrm{D}+\mathrm{T}$ or ipi/nivo followed by a switch to the other combination at progression), may be robust tools for comparing efficacy; but these approaches also have limitations in their ability to evaluate outcomes across heterogeneous patient and treatment populations or in being contemporaneous with evolving treatment patterns. Real-world observational research is one methodological approach for addressing these gaps; however, at the time of this writing, few realworld studies had compared outcomes between TT and IO for BRAF mutant MM patients since the introduction of PD-1 treatments [9-14]. The aims of this research were to describe characteristics of real-world BRAF mutant 
MM patients prescribed 1L TT or IO, as well as the duration of $1 \mathrm{~L}$ therapy, time between discontinuation of $1 \mathrm{~L}$ to initiation of second-line (2L) therapy, disease response and overall survival (OS).

\section{Materials \& methods}

\section{Study design}

This was a retrospective, observational study of BRAF mutant MM patients treated with 1L TT or IO therapy conducted using a chart review methodology. The preplanned study sample size was 600 patients. Providers selected a convenience sample of patients meeting the following inclusion criteria: confirmed BRAF V600 activating mutation; initiated 1L therapy after 1 January 2014 and before 30 June 2017; received $1 \mathrm{~L}$ therapy with D+T, V+C, ipi, ipi/nivo, nivo or pembro; completed at least one cycle of $1 \mathrm{~L}$ therapy; and followed for at least 6 months from the date of treatment initiation (or until death). Patients $<18$ years of age at the initiation of $1 \mathrm{~L}$ therapy, who received $1 \mathrm{~L}$ therapy as part of a clinical trial, or who were diagnosed with a second primary cancer following initiation of $1 \mathrm{~L}$ therapy, were not eligible.

\section{Data collection}

Data were collected through a chart review by providers in the Cardinal Health Oncology Provider Extended Network (OPEN), an informal community of over 7000 oncologists from across the USA. Providers identified patients meeting the selection criteria and abstracted data in an anonymized electronic case report form (eCRF). For all data points providers were given the option to select 'unknown' or 'data not collected' if the data were unavailable in the patient's record. Data collection completed on 1 October 2018. The study protocol and data capture tool were reviewed by a central institutional review board who provided a waiver of obtaining informed consent from patients. Data from a random selection of $10 \%$ of cases were verified by requiring physicians to re-enter select data points at a later date.

\section{Analyses}

The primary study cohorts for analysis were patients treated in $1 \mathrm{~L}$ with any combination $\mathrm{TT}(\mathrm{D}+\mathrm{T}$ or $\mathrm{V}+\mathrm{C})$ versus any $1 \mathrm{~L}$ IO therapy (ipi/nivo, nivo, pembro). Additionally, subgroup comparisons were made by treatment regimen (D+T vs ipi/nivo; $\mathrm{D}+\mathrm{T}$ vs PD-1 monotherapy), in an attempt to further understand comparative effectiveness between TT and IO therapies. The PD-1 monotherapy group included any patients treated with either singleagent nivo or pembro. Comparisons of the $\mathrm{V}+\mathrm{C}$ arm to $\mathrm{IO}$ therapies were not made owing to the similarities in mechanism of action to $\mathrm{D}+\mathrm{T}$ and the comparatively smaller sample size.

Treatment sequences in $1 \mathrm{~L}$ and $2 \mathrm{~L}$, time on $1 \mathrm{~L}(T O n T)$, time from discontinuing $1 \mathrm{~L}$ to initiating $2 \mathrm{~L}$ (time off treatment [ToffT]), disease response and OS were measured. TOnT was the interval from initiation of $1 \mathrm{~L}$ until discontinuation of $1 \mathrm{~L}$ due to any reason. Toff T was the interval from discontinuation of $1 \mathrm{~L}$ treatment until initiation of $2 \mathrm{~L}$ treatment. Patients on $1 \mathrm{~L}$ treatment at the end of the study period were censored on the last date of known treatment. Disease response was assessed in two ways: provide reported-using the response description in the medical record; and retrospectively calculated based on RECIST v1.1 guidelines using lesion measurements provided by abstractors [15]. Provider-reported disease response was based on language used within the medical record narrative; providers were asked to indicate the patients' best response to $1 \mathrm{~L}$ therapy as complete response (CR), partial response (PR), stable disease (SD) or progressive disease (PD). Retrospectively calculated response according to RECIST guidelines was performed by having providers abstract lesion measurements from CT, PET and/or other imaging at initiation of $1 \mathrm{~L}$ treatment and at time of best response to $1 \mathrm{~L}$. Using these lesion measurements (to determine target lesions) as reported by the provider (and asking providers to report whether new lesions had appeared), disease response (CR, PR, SD or PD) was calculated based on RECIST v1.1 guidelines. No independent or central review of radiology images was conducted. The objective response rate (ORR) was taken as the sum of CRs and PRs. Rates of response in the retrospective calculation are based on the total patient sample, including those patients for which response could not be assessed (best response not yet occurred or unavailability of measurements of baseline lesions at best response). OS was defined as the interval from the initiation of $1 \mathrm{~L}$ therapy until death due to any cause. Patients alive at the end of study period were censored on the last known date of follow-up.

Descriptive statistics were performed, and univariate comparisons of categorical and continuous variables were made using $\chi^{2}$ tests and t-tests. Unknown ormissing data are shown as occurred; for continuous measures only patients with known values were considered. TOnT, ToffT and OS were estimated using the Kaplan-Meier technique 
with statistical comparison via the log-rank test, as not all patients had completed $1 \mathrm{~L}$ treatment (right-censored). A hierarchical logistic regression model was used to examine likelihood of an objective disease response via RECIST v1.1, comparing cohorts and subgroups, including patients with unknown/not evaluable best disease response tumor measurements accounting for nesting of patients among providers [16]. Multivariate Cox-proportional hazards models were used to compare the risk of treatment discontinuation (from the TOnT estimates) between cohorts and subgroups. The risk of treatment discontinuation was the likelihood of the patient discontinuing $1 \mathrm{~L}$ treatment for any reason (e.g., progression, death, toxicity, patient choice, etc.) over the interval. Both the logistic regression model and the Cox models were adjusted for age $(<65, \geq 65)$, sex, presence of brain metastases, presence of liver metastases, total number of distant metastatic sites $(1-3, \geq 4)$ ECOG-PS $(0 / 1$ or $\geq 2)$ and lactate dehydrogenase (LDH; normal or abnormal). Actual LDH values were not collected in the eCRF; providers indicated whether the patient's LDH was normal, abnormal and $<$ two-times the upper limit of normal, or abnormal and $\geq$ two-times the upper limit of normal based upon charted lab reference range.

\section{Results}

Patients' cohorts \& selection of $1 \mathrm{~L}$ treatment

A total of 53 providers $(60.3 \%$ practicing in the northeast, $15.1 \%$ midwest, $13.2 \%$ south and $11.3 \%$ west) participated in the study and contributed data on 440 patients with BRAF mutant MM who met the inclusion criteria. Recruitment was stopped at 440 out of the planned 600, as providers reported no further patients could be identified. The final analytical cohort consisted of 283 patients treated in $1 \mathrm{~L}$ with TT (61.5\%) and 157 patients treated with IO (34.1\%). By individual treatment regimens received in $1 \mathrm{~L}$, there were: $\mathrm{D}+\mathrm{T}=188, \mathrm{~V}+\mathrm{C}=95$, ipi $/$ nivo $=81$, PD-1 mono $=76$.

Demographic and clinical characteristics of the TT and IO cohorts and the subgroups are summarized in Table 1. Both TT- and IO-treated patients were predominantly male $(59.4 \%$ vs $58.6 \%)$ and covered by commercial/employer sponsored insurance $(52.7 \%$ vs $46.5 \%)$. The median age at $1 \mathrm{~L}$ treatment initiation was 61 years in both cohorts. Regarding disease characteristics, the TT cohort had significantly higher proportions of patients with liver metastases ( 46.3 vs $35.0 \% ; \mathrm{p}=0.02$ ) and abnormal LDH $(61.1$ vs $42.7 \%$; $\mathrm{p}<0.01$ ) and significantly lower proportion of patients with any comorbidities ( 37.0 vs $48.0 \% ; \mathrm{p}=0.03)$. Between the subgroups, patients treated with $1 \mathrm{~L} \mathrm{D}+\mathrm{T}$ were significantly older $(60.8 \mathrm{vs} 56.5 ; \mathrm{p}=0.01)$ than those treated with $1 \mathrm{~L}$ ipi/nivo, but significantly younger than those treated with PD-1 monotherapy (60.8 vs 66.3; p < 0.01) (Table 1). Patients treated with $1 \mathrm{~L} \mathrm{D}+\mathrm{T}$ also had less favorable distributions with respect to total number of distant metastatic sites and LDH levels, relative to those treated with $1 \mathrm{~L}$ PD-1 monotherapy. At end of follow-up, $17.3 \%(\mathrm{n}=49)$ of patients in the TT cohort and $15.9 \%(\mathrm{n}=25)$ of patients in the IO cohort were deceased, with an additional $1.8 \%$ and $5.1 \%$, respectively, actively receiving hospice care. No statistically significant differences in number of lines of therapy, deaths or length of follow-up were noted between the main cohorts and the subgroups.

Treatment sequences are displayed in Table 2. Among the 97 patients receiving $\mathrm{D}+\mathrm{T}$ in $1 \mathrm{~L}$ and receiving a $2 \mathrm{~L}$ treatment, $59.8 \%$ received PD-1 monotherapy as $2 \mathrm{~L}$. Among the 50 patients receiving ipi/nivo and 43 patients receiving PD-1 monotherapy in $1 \mathrm{~L}$ and receiving $2 \mathrm{~L}$ treatment, $62.0 \%$ and $44.2 \%$, respectively, received $2 \mathrm{~L} \mathrm{D}+\mathrm{T}$.

\section{Time on treatment, time off treatment \& OS}

TOnT was significantly longer for patients treated with TT in $1 \mathrm{~L}$ compared with those treated with IO in $1 \mathrm{~L}(11.4$ vs 7.2 months; Figure 1A - TOnT), driven by the short duration for patients treated with ipi/nivo (4.6 months; Figure $1 \mathrm{~B}-\mathrm{TOnT})$. No difference in TOnT was noted between $\mathrm{D}+\mathrm{T}$ and $\mathrm{PD}-1$ monotherapy $(\mathrm{p}=0.25$, Figure $1 \mathrm{C}$ - TOnT). ToffT was significantly longer for patients treated with IO (1.5 vs 0.6 months; Figure $1 \mathrm{~A}$ - ToffT), driven by long ToffT of patients treated with ipi/nivo (4.6 months; Figure 1B - ToffT). The risk of treatment discontinuation was significantly higher among patients treated with IO therapy compared with TT $(\mathrm{HR}=1.64$; 95\% CI: 1.25-2.14; $\mathrm{p}<0.001)$ and was three times higher comparing ipi/nivo to $\mathrm{D}+\mathrm{T}$ ( $\mathrm{HR}=3.24 ; 95 \% \mathrm{CI}$ : 2.26-4.64; $\mathrm{p}<0.001)$. There was no significant difference in the risk of discontinuation between $\mathrm{D}+\mathrm{T}$ and $\mathrm{PD}-1$ monotherapy ( $\mathrm{HR}=1.00 ; 95 \% \mathrm{CI}: 0.68-1.49 ; \mathrm{p}=0.99)$. Median OS from 1L initiation of TT was 33.1 months (95\% CI: 26.1-37.1 months) and was not reached in the IO-treated cohort. Median OS was not significantly different between the cohorts $(\mathrm{p}=0.664)$. 


\begin{tabular}{|c|c|c|c|c|c|}
\hline Demographics & $\pi(n=283)$ & $10(n=157)$ & $D+T(n=188)$ & ipi /nivo $(n=86)$ & PD-1 mono $(n=71)$ \\
\hline Male & $168(59.4 \%)$ & $92(58.6 \%)$ & $115(61.2 \%)$ & $53(61.6 \%)$ & $39(54.9 \%)$ \\
\hline Mean age at treatment initiation (median) & $60.2(61 \%)$ & $60.9(61 \%)$ & $60.8(61 \%)$ & $56.5(55.5 \%)$ & $66.3(69 \%)$ \\
\hline \multicolumn{6}{|l|}{ Payer $^{\dagger}:$} \\
\hline - Medicare & $55(19.4 \%)$ & $39(24.9 \%)$ & $42(22.3 \%)$ & $12(14.0 \%)$ & $27(38.0 \%)$ \\
\hline - Medicare advantage/supplemental & $60(21.2 \%)$ & $36(22.9 \%)$ & $38(20.2 \%)$ & $15(17.4 \%)$ & $21(29.6 \%)$ \\
\hline - Medicaid & $25(8.8 \%)$ & $13(8.3 \%)$ & $17(19.0 \%)$ & $11(12.8 \%)$ & $2(2.8 \%)$ \\
\hline - Commercial/employer-sponsored & $149(52.7 \%)$ & $73(46.5 \%)$ & $98(52.1 \%)$ & $49(57.0 \%)$ & $24(33.8 \%)$ \\
\hline - Other/unknown & $6(2.1 \%)$ & $8(5.1 \%)$ & $3(1.6 \%)$ & $5(5.8 \%)$ & $3(4.2 \%)$ \\
\hline \multicolumn{6}{|l|}{ M-stage at diagnosis: } \\
\hline - M0, M1a, M1b & $114(40.3 \%)$ & $76(48.4 \%)$ & $71(37.8 \%)$ & $39(45.3 \%)$ & $37(52.1 \%)$ \\
\hline$-\mathrm{M} 1 \mathrm{c}$ & $169(59.7 \%)$ & $81(51.6 \%)$ & $117(62.2 \%)$ & $47(54.7 \%)$ & $34(47.9 \%)$ \\
\hline \multicolumn{6}{|l|}{ Site of metastases ${ }^{\dagger}:$} \\
\hline - Lung & $192(67.8 \%)$ & $110(70.1 \%)$ & $131(69.7 \%)$ & $58(67.4 \%)$ & $52(73.2 \%)$ \\
\hline - Liver & $131(46.3 \%)$ & $55(35.0 \%)$ & $85(45.2 \%)$ & $32(37.2 \%)$ & $23(32.4 \%)$ \\
\hline - Brain & $28(9.9 \%)$ & $15(9.6 \%)$ & $16(8.5 \%)$ & $7(8.1 \%)$ & $8(11.3 \%)$ \\
\hline - Bone & $47(16.6 \%)$ & $25(15.9 \%)$ & $30(16.0 \%)$ & $18(20.9 \%)$ & $7(9.9 \%)$ \\
\hline - Lymph nodes & $65(23.0 \%)$ & $42(26.8 \%)$ & $43(22.9 \%)$ & $23(26.7 \%)$ & $19(26.8 \%)$ \\
\hline - Subcutaneous tissue & $32(11.3 \%)$ & $14(8.9 \%)$ & $19(10.1 \%)$ & $4(4.7 \%)$ & $10(14.1 \%)$ \\
\hline - Other & $15(5.3 \%)$ & $7(4.5 \%)$ & $9(4.8 \%)$ & $4(4.7 \%)$ & $3(4.2 \%)$ \\
\hline \multicolumn{6}{|l|}{ Total number of distant metastatic sites at initiation of $1 \mathrm{~L}$ : } \\
\hline$-1-3$ & $229(80.9 \%)$ & $131(83.4 \%)$ & $148(78.7 \%)$ & $67(77.9 \%)$ & $64(90.1 \%)$ \\
\hline$-\geq 4$ & $54(19.0 \%)$ & $26(16.6 \%)$ & $40(21.3 \%)$ & $19(22.1 \%)$ & $7(9.9 \%)$ \\
\hline \multicolumn{6}{|l|}{ ECOG-PS at metastatic diagnosis: } \\
\hline$-0 / 1$ & $245(86.6 \%)$ & $129(82.2 \%)$ & $158(84.0 \%)$ & $77(89.5 \%)$ & $52(73.2 \%)$ \\
\hline$-\geq 2$ & $38(13.4 \%)$ & $28(17.8 \%)$ & $30(16.0 \%)$ & $9(10.5 \%)$ & $19(26.8 \%)$ \\
\hline \multicolumn{6}{|l|}{$\operatorname{LDH}(n, \%):$} \\
\hline - Normal & $103(36.4 \%)$ & $81(51.6 \%)$ & $73(38.8 \%)$ & $39(45.3 \%)$ & $42(59.2 \%)$ \\
\hline - Abnormal & $173(61.1 \%)$ & $67(42.7 \%)$ & $111(59.0 \%)$ & $44(51.2 \%)$ & $23(32.4 \%)$ \\
\hline - Unknown & $7(2.5 \%)$ & $9(5.7 \%)$ & $4(2.1 \%)$ & $3(3.5 \%)$ & $6(8.5 \%)$ \\
\hline Any comorbidity $(n, \%)$ & $133(47.0 \%)$ & $91(58.0 \%)$ & $97(51.6 \%)$ & $47(54.7 \%)$ & $44(62.0 \%)$ \\
\hline $\begin{array}{l}\text { Mean months of follow-up from } 1 \mathrm{~L} \text { treatment initiation } \\
\text { (median) }\end{array}$ & $14.1(12.4 \%)$ & $14.5(13.05)$ & $14.3(12.4 \%)$ & $14.1(12.5 \%)$ & $15.0(13.1 \%)$ \\
\hline \multicolumn{6}{|l|}{ Total lines of therapy $(n, \%)$ : } \\
\hline-1 & $141(49.8 \%)$ & $64(40.8 \%)$ & $91(48.4 \%)$ & $36(41.9 \%)$ & $28(39.4 \%)$ \\
\hline-2 & $123(43.5 \%)$ & $84(53.5 \%)$ & $85(45.2 \%)$ & $44(51.2 \%)$ & $40(56.3 \%)$ \\
\hline$-\geq 3$ & $19(6.7 \%)$ & $9(6.3 \%)$ & $12(6.3 \%)$ & $6(7.0 \%)$ & $3(4.2 \%)$ \\
\hline
\end{tabular}

\section{Table 2. Treatment patterns in first-line and second-line.}

First-line treatment regimen (number receiving second-line)

Second-line treatment regimen, number receiving (row \%)

\begin{tabular}{|c|c|c|c|c|c|c|c|}
\hline & & & & & & & \\
\hline & $D+T$ & $\mathrm{~V}+\mathrm{C}$ & ipi/nivo & PD-1 mono & ipi & Single-agent TT & Other \\
\hline \multirow{2}{*}{$D+T(n=97)$} & 0 & 4 & 21 & 58 & 7 & 2 & 5 \\
\hline & $0.0 \%$ & $4.1 \%$ & $21.6 \%$ & $59.8 \%$ & $7.2 \%$ & $2.1 \%$ & $5.2 \%$ \\
\hline \multirow[t]{2}{*}{ ipi/nivo $(n=50)$} & 31 & 10 & 0 & 3 & 0 & 3 & 2 \\
\hline & $62.0 \%$ & $20.0 \%$ & $0.0 \%$ & $6.0 \%$ & $0.0 \%$ & $6.0 \%$ & $4.0 \%$ \\
\hline \multirow[t]{2}{*}{ PD-1 mono $(n=43)$} & 19 & 10 & 2 & 3 & 1 & 8 & 0 \\
\hline & $44.2 \%$ & $23.3 \%$ & $4.7 \%$ & $7.0 \%$ & $2.3 \%$ & $18.6 \%$ & $0.0 \%$ \\
\hline
\end{tabular}

ipi: Ipilimumab; nivo: Nivolumab; TT: Targeted therapy. 

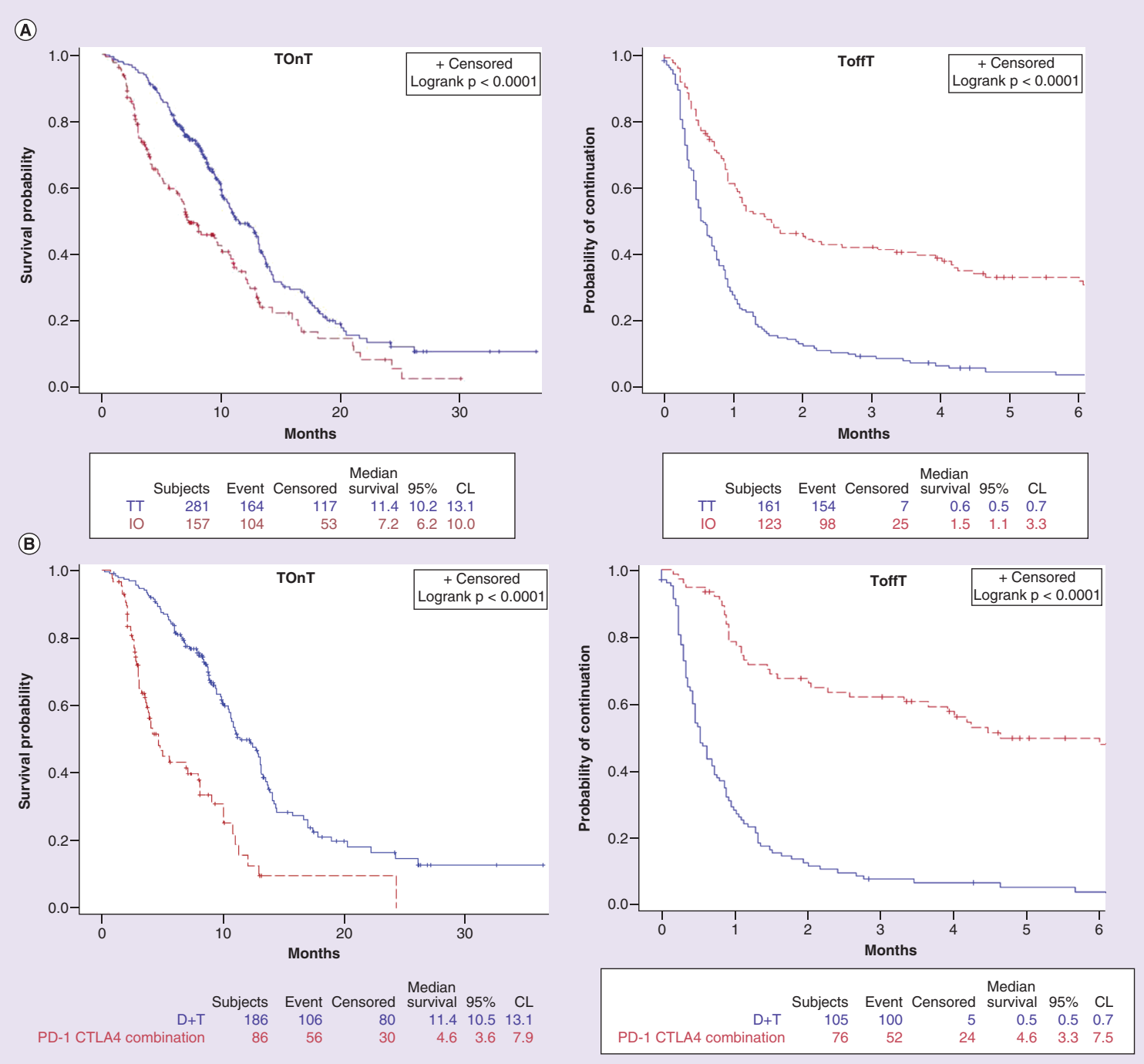

Figure 1. TOnT and ToffT for (A) TT vs IO therapy, (B) D+T vs ipi/nivo, and (C) D+T vs PD-1 monotherapy. IO: Immuno-oncology; ipi: Ipilimumab; nivo: Nivolumab; TT: Targeted therapy.

\section{Disease response during $1 \mathrm{~L}$ therapy}

Response to $1 \mathrm{~L}$ therapy, both provider-reported and retrospectively measured using RECIST v1.1 criteria are summarized in Table 3. The ORR, based on provider report, was $79.2 \%$ for $1 \mathrm{~L} \mathrm{TT}$, compared with $71.3 \%$ for $1 \mathrm{~L}$ IO ( $p=0.06)$. Rates of CR, PR, SD, and PD were significantly different between TT and IO $(p=0.01)$.

The retrospectively calculated disease response rates using RECIST v1.1 criteria were directionally similar to the rates based on provider report, but were of a smaller absolute difference, except the $56.2 \%$ PR rate for TT-treated compared to $40.8 \%$ for IO-treated among those patients with measurable lesions (Table 3). The ORR for TT was 60.1 vs $45.9 \%$ for IO ( $\mathrm{p}<0.01$ ); the ORR for $\mathrm{D}+\mathrm{T}$ was $60.6 \%$, significantly greater than the $42.3 \%$ reported for PD-1 monotherapy $(\mathrm{p}=0.01)$. Comparing the two methods of disease response measurement, the overall concordance between provider-reported versus RECIST-calculated was $75.9 \%(277 / 365)$.

In the adjusted model, odds of objective response in 1L (via RECIST) were $49 \%$ lower for IO compared with TT (odds ratio $=0.51 ; 95 \%$ CI: $0.32-0.81 ; \mathrm{p}=0.004), 60 \%$ lower for those treated with PD-1 monotherapy compared with $\mathrm{D}+\mathrm{T}$ (odds ratio $=0.40 ; 95 \% \mathrm{CI}: 0.21-0.74 ; \mathrm{p}=0.004$ ), and $45 \%$ lower for patients treated with ipi $/$ nivo versus $\mathrm{D}+\mathrm{T}$ (odds ratio $=0.55 ; 95 \% \mathrm{CI}$ : $0.30-1.00 ; \mathrm{p}=0.05$ ). 

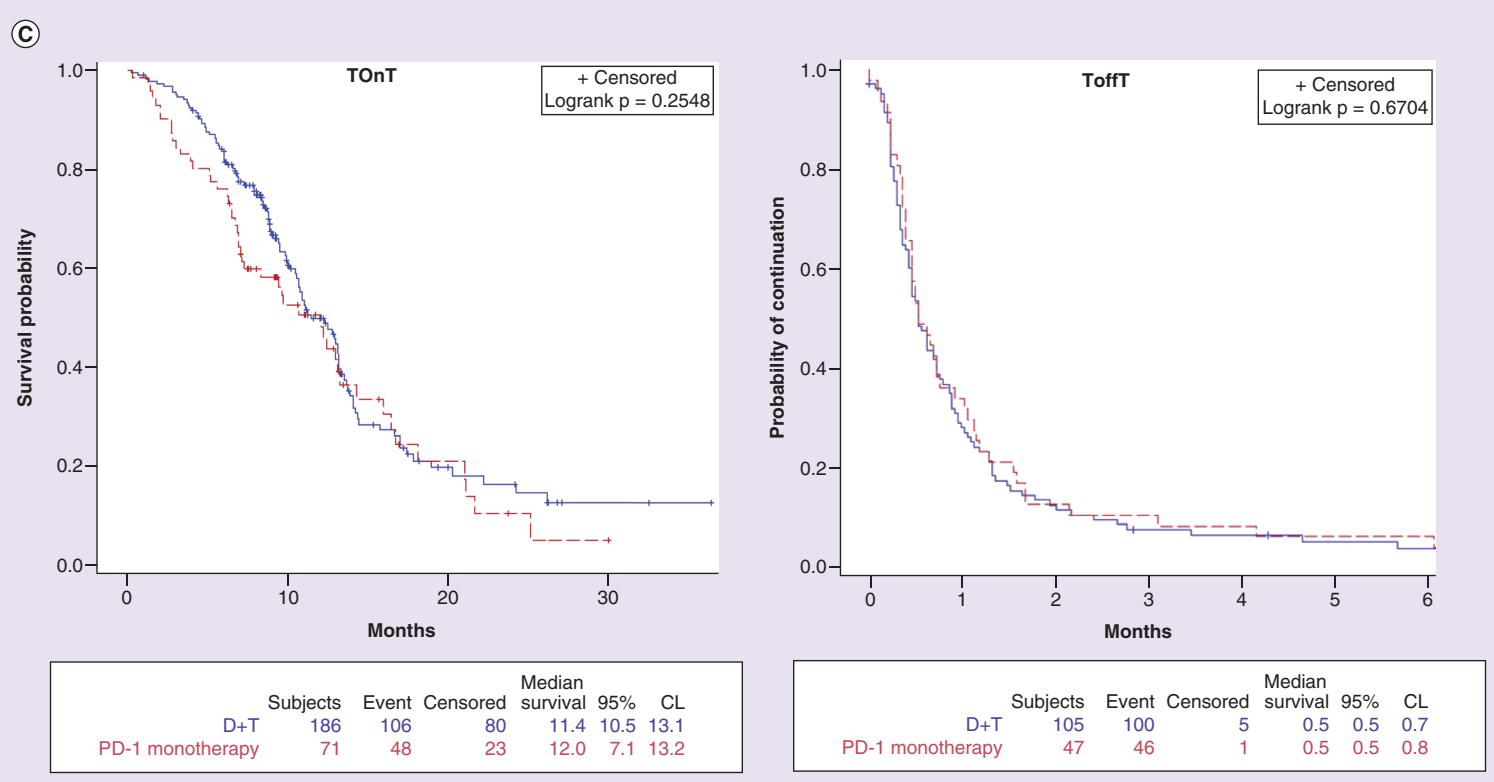

Figure 1. TOnT and ToffT for (A) TT vs IO therapy, (B) D+T vs ipi/nivo, and (C) D+T vs PD-1 monotherapy (cont.).

IO: Immuno-oncology; ipi: Ipilimumab; nivo: Nivolumab; TT: Targeted therapy.

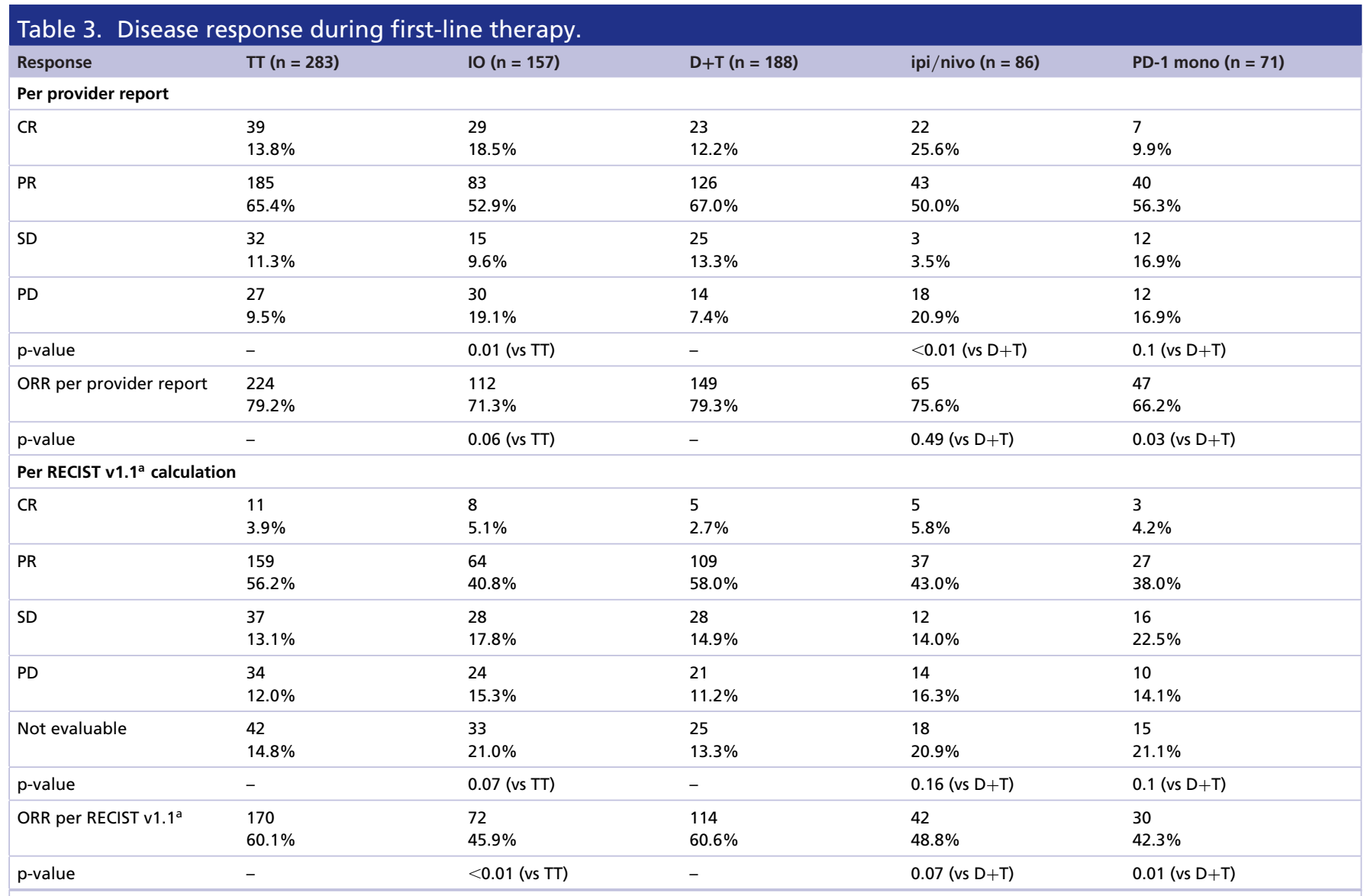

CR: Complete response; IO: Immuno-oncology; ipi: Ipilimumab; nivo: Nivolumab; ORR: Objective response rate; PD: Progressive disease; PR: Partial response; SD: Stable disease; TT: Targeted therapy. 


\section{Discussion}

To investigate outcomes of $1 \mathrm{~L}$ treatment for BRAF mutant MM patients, we described and compared real-world patients receiving $1 \mathrm{~L}$ TT or IO, their disease response, the duration of response through the surrogates of TOnT and ToffT and OS. We observed an apparent provider preference for TT as $1 \mathrm{~L}$ treatment in real-world BRAF mutant MM patients. This appeared to be especially the case for patients with higher risk disease at diagnosis as indicated by a higher proportion of patients in the TT-cohort with liver metastases and an elevated LDH. This real-world finding may be of particular importance to the optimal application of TT surrounding optimal application of TT, given clinical trial evidence that patients with low $\mathrm{LDH}$ derive longer-term benefit from $\mathrm{D}+\mathrm{T}$ [17].

Although overall follow-up was short, nearly half of patients $(n=207)$ received $2 \mathrm{~L}$ therapy. We observed a longer TOnT for TT versus IO therapy; however, the median ToffT was significantly longer for IO-treated patients at 1.5 months versus 0.6 months for TT-treated. This was driven by the 4.6-month average ToffT for ipi/nivotreated patients prior to initiating $2 \mathrm{~L}$ [18]. Given the short ToffT for TT-treated patients, TOnT may be a close approximation to progression free survival (PFS) in the TT-treated cohort. TOnT among TT-treated patients was 11.4 months which is consistent, if slightly shorter, than the median PFS observed in the D+T trials (12.6 months), and equivalent to that of the $\mathrm{V}+\mathrm{C}$ trials (11.4 months) [19]. For the IO-treated patients, the observed median PFS in CheckMate 067 was 11.5 months in the ipi/nivo arm. Our results, considering both the TOnT (at 4.6 months) and the ToffT (4.6 months), show a slightly shorter PFS interval for ipi/nivo-treated patients. For patients treated with PD-1 monotherapies, we observed a TOnT nearly double (12.0 months) the median PFS estimates observed in both CheckMate 067 (6.9 months) [5] and post hoc analyses of treatment-naive pembro-treated patients in KEYNOTE-006 (6.2 months with only 26.6\% of patients BRAF-mutated) [20].

Regarding long-term outcomes, the median OS of all patients was 37.1 months (74 death events); however, we were unable to assess OS by therapeutic sequencing given the relatively short median duration of follow-up in the cohort $(\mathrm{TT}=14.5$ months, $\mathrm{IO}=14.1$ months from initiation of $1 \mathrm{~L}$ ). The OS observed in our study was within the range of estimates from respective RCTs, but further follow-up on par with those trials is needed and may lead to different conclusions [17-19,21]. A recent study of patients who received sequential BRAF inhibitor followed by ipi demonstrated a median OS of 28 months [14]. Comparisons of IO trial results to our study are difficult given a lack of any post hoc published estimates of BRAF-mutated subgroup outcomes analyses. Further research, such as the ECOG-ACRIN trial (NCT02224781), will hopefully answer many of these outstanding questions regarding how treatment sequence impacts OS.

Finally, we found both provider-reported and retrospectively measured disease response rates higher among patients treated with TT than IO in 1L. The ORR for TT was significantly higher by retrospective classification according to the RECIST criteria $(\mathrm{p}<0.01)$ but not provider-reported $(\mathrm{p}=0.06)$. Of note, the RECIST calculated rates were approximately $20 \%$ lower than those in the medical record as reported by providers. The response benefit persisted after adjusting for baseline clinical differences (odds of response for IO- versus TT-treated $=0.52$; 95\% CI: $0.34-0.80 ; \mathrm{p}<0.01)$. In comparison, a retrospective analysis of 114 patients with $\mathrm{MM}$ who had received PD-1 treatments either before or after a BRAF inhibitor alone, or with a MEK inhibition, found RECIST-based response rates to $1 \mathrm{~L}$ PD- 1 therapy of $41 \%$, compared with $46 \%$ observed in our population (the corresponding ORR to 1L BRAF-mutated TT was not reported) [11]. A recent analysis exploring differences in response between BRAFv600K and v600E found patients with v600K mutations had greater mutational burden and derived greater benefit from IO compared with TT which may explain differences should ourpopulation of patients had higher proportion of v600E mutations [22]. In CheckMate 067 the ORR for ipi/nivo-treated patients was 58 and 44\% in the nivo-treated arm, which is approximately $10 \%$ higher compared with our study [5]. We note that a major difference is that CheckMate 067 includes a significant proportion of BRAF wild-type patients (68\%) for which response to IO therapy may be higher. Comparing ORRs for D+T-treated patients to RCTs, we found greater consistency. The ORR in our study for $\mathrm{D}+\mathrm{T}$-treated patients was also lower at $61 \%$ compared with 69 and $64 \%$ in the COMBI-d and COMBI-v trials, respectively $[2,23]$. Altogether these findings reinforce the idea that the diversity of patients treated in the real-world requires a treatment decision based on a comprehensive evaluation of the goals of treatment, the clinical characteristics of the patients and likelihood of toxicity.

There are several important limitations that the authors acknowledge: first, providers were not randomly selected and cases were identified by providers and not all eligible patients treated by the providers may have been included (convenience sample), resulting in a possible selection bias. Second, providers entered data manually into the eCRF which may result in transcription errors (e.g., incorrect dates). We attempted to minimize these errors through 
eCRF validation; however, only $10 \%$ (randomly selected) of charts were validated. Next, date of death was per provider record in the patient's chart and not based on mapping of patients to external databases such as the social security death index master file. Fourth, LDH levels were not collected. It was assumed that providers classified the patient's LDH value based on the upper limits provided by the laboratory at the time of reporting; however, this could not be verified as source laboratory reports were not evaluated. Finally, the rationale for the selection of TT versus IO therapy for each individual patient was not ascertained. Unmeasured confounding due to insurance coverage, patient choice or other undocumented clinical factors may have existed. However in adjusted analyses, we attempted to control for these factors by using a hierarchical model which simultaneously controls for provider factors, patient demographics, and clinical characteristics..

The major strengths of this research were the size of the study population (which to our knowledge is the largest real-world, US-based cohort study of BRAF mutant MM), its contemporaneity (last follow-up January 2018), and the evaluation of both provider-reported and objective (albeit retrospective) assessment of disease response per RECIST v1.1. Overall, our findings emphasize a comfort level for providers to treat BRAF mutant MM patients consistent with guidelines recommending TT for patients needing rapid disease response. Approaches utilizing IO appeared to be more likely chosen in patients with a more favorable clinical profile, potentially indicating a greater ability to tolerate those therapies. With no observed difference in OS, the selection of a $1 \mathrm{~L}$ treatment for BRAF mutant MM patients requires a critical evaluation of the disease burden and likelihood of toxicity.

\section{Conclusion}

Community oncologists in the US may prefer 1L TT for BRAF mutant MM, especially in higher-risk patients with liver metastases and elevated LDH. Combination TT was associated with a significantly higher ORR and significantly longer TOnT than 1L IO therapy and may be explained by differences in the mutational burden of patients. Our OS estimates were longer than those previously reported for $\mathrm{D}+\mathrm{T}$ in clinical trials, and median $\mathrm{OS}$ was not reached among patients treated with IO therapy. However, no significant differences in OS were observed.

Financial \& competing interests disclosure

This study was sponsored and funded by Novartis Pharmaceuticals Corporation East Hanover, New Jersey. The authors are fully responsible for all content and editorial decisions.

J Luke: Data and Safety Monitoring Board: TTC Oncology; Scientific Advisory Board: 7 Hills, Actym, Alphamab Oncology, Array, BeneVir, Mavu; Consultancy: Aduro, AstraZeneca, Bristol- Myers Squibb, Castle, CheckMate, Compugen, EMD Serono, Ideaya, Janssen, Merck, NewLink, Novartis, RefleXion, Spring Bank, Syndax, Tempest, Vividion, WntRx; Research Support: (all to institution for clinical trials unless noted) AbbVie, Array (Scientific Research Agreement; SRA), Boston Biomedical, Bristol-Myers Squibb, Celldex, CheckMate (SRA), Compugen, Corvus, EMD Serono, Evelo (SRA), Delcath, Five Prime, Genentech, Immunocore, Incyte, Leap, Medlmmune, Macrogenics, Novartis, Pharmacyclics, Palleon (SRA), Merck, Tesaro, Xencor; Travel: Array, AstraZeneca, BeneVir, Bristol-Myers Squibb, Castle, CheckMate, EMD Serono, Ideaya, Janssen, Merck, NewLink, Novartis, RefleXion; Patents: (both provisional) Serial \#15/612,657 (Cancer Immunotherapy), PCT/US18/36052 (Microbiome Biomarkers for Anti-PD-1/PD-L1 Responsiveness: Diagnostic, Prognostic and Therapeutic Uses Thereof);

J Kish, C Lee, L McAllister, S Mehta and B Feinberg are employed by Cardinal Health Specialty Solutions; and S Ghate and B Ndife are employed by Novartis. The authors have no relevant affiliations or financial involvement with any organization or entity with a financial interest in or financial conflict with the subject matter or materials discussed in the manuscript apart from those disclosed.

The authors acknowledge Laurie Orloski for medical writing support.

\section{Ethical conduct}

The authors state that they have obtained appropriate institutional review board approval or have followed the principles outlined in the Declaration of Helsinki for all human or animal experimental investigations. In addition, for investigations involving human subjects, informed consent has been obtained from the participants involved.

\section{Open access}

This work is licensed under the Attribution-NonCommercial-NoDerivatives 4.0 Unported License. To view a copy of this license, visit http://creativecommons.org/licenses/by-nc-nd/4.0/ 
- Seven drugs, including targeted therapy (TT) and immunotherapy agents, were approved by the US FDA for treating metastatic BRAF mutant metastatic melanoma (MM) over a 4-year period.

- Clinical decision-making for these patients is hampered by a lack of head-to-head randomized controlled trials comparing TT and IO for BRAF mutant MM and a paucity of real-world observational research.

- This retrospective, observational study of BRAF mutant MM patients, conducted using a chart review methodology, evaluated characteristics of BRAF mutant MM patients prescribed $1 \mathrm{~L}$ TT or IO, the duration of first-line therapy (1L), time to second-line therapy (2L), the extent and duration of treatment response and overall survival (OS)

- A total of 440 patients were analyzed. The TT cohort had significantly higher proportions of patients with liver metastases (46.3 vs $35.0 \%$ ) and abnormal lactate dehydrogenase (61.1 vs $42.7 \%$ ).

- Provider-reported objective response rates were $79.2 \%$ for $1 \mathrm{~L}$ TT versus $71.3 \%$ for $1 \mathrm{~L}$ IO $(p=0.06)$; per RECIST v1.1 criteria, corresponding rates were 60.1 versus $45.9 \%$, respectively $(p<0.01)$. Per an adjusted model, odds of objective response in $1 \mathrm{~L}$ (via RECIST) were $49 \%$ lower for IO versus TT ( $p=0.004)$.

- For $1 \mathrm{~L}$, time on treatment was significantly longer for patients treated with TT versus IO (11.4 vs 7.2 months) whereas time off treatment (calculated from discontinuation of $1 \mathrm{~L}$ to the initiation of $2 \mathrm{~L}$ ) was significantly longer for patients treated with 10 versus TT (1.5 vs 0.6 months).

- Median OS from $1 \mathrm{~L}$ initiation of TT was 33.1 months (95\% Cl: 26.1-37.1 months) and was not reached with IO, with no statistical significance between the cohorts.

- Overall, these results suggest that providers may have prioritized treatment of patients with BRAF mutant MM-consistent with guidelines recommending TT for patients needing rapid disease response.

- With no observed difference in OS, the selection of a $1 \mathrm{~L}$ treatment for a BRAF mutant MM patient requires a critical evaluation of the disease burden and likelihood of toxicity.

\section{References}

Papers of special note have been highlighted as: $\bullet$ of interest; $\bullet \bullet$ of considerable interest

1. National Comprehensive Cancer Network (NCCN). NCCN Clinical Practice Guidelines in Oncology (NCCN Guidelines): melanoma. Version 2.2018- January 19 2018. https://www.nccn.org/patients/guidelines/melanoma/

2. Long GV, Stroyakovskiy D, Gogas H et al. Dabrafenib and trametinib versus dabrafenib and placebo for Val600 BRAF-mutant melanoma: a multicentre, double-blind, Phase III randomised controlled trial. Lancet 386(9992), 444-451 (2015).

3. Larkin J, Del Vecchio M, Ascierto PA et al. Vemurafenib in patients with BRAF(V600) mutated metastatic melanoma: an open-label, multicentre, safety study. Lancet Oncol. 15(4), 436-444 (2014).

4. Larkin J, Ascierto PA, Dreno B et al. Combined vemurafenib and cobimetinib in BRAF-mutated melanoma. N. Engl. J. Med. 371(20), 1867-1876 (2014).

5. Larkin J, Chiarion-Sileni V, Gonzalez R et al. Combined nivolumab and ipilimumab or monotherapy in untreated melanoma. N. Engl. J. Med. 373(1), 23-34 (2015).

6. Robert C, Schachter J, Long GV et al. Pembrolizumab versus ipilimumab in advanced melanoma. N. Engl. J. Med. 372(26), 2521-2532 (2015).

7. Dummer R, Ascierto PA, Gogas HJ et al. Encorafenib plus binimetinib versus vemurafenib or encorafenib in patients with BRAF-mutant melanoma (COLUMBUS): a multicentre, open-label, randomised Phase III trial. Lancet Oncol. 19(5), 603-615 (2018).

8. Ascierto PA, Caraco C, Gershenwald JE et al. The Great Debate at 'Melanoma Bridge', Napoli, December 2nd, 2017. J. Transl. Med. 16(1), 101 (2018).

9. Ackerman A, Klein O, McDermott DF et al. Outcomes of patients with metastatic melanoma treated with immunotherapy prior to or after BRAF inhibitors. Cancer 120(11), 1695-1701 (2014).

- This retrospective analysis of 274 patients receiving a BRAF inhibitor (alone or with MEK inhibition) before or after immunotherapy provides some insight into the sequencing, with poor outcomes for immunotherapy following BRAF inhibition but no negative influence of immunotherapy on response to BRAF inhibition.

10. Ascierto PA, Simeone E, Sileni VC et al. Sequential treatment with ipilimumab and BRAF inhibitors in patients with metastatic melanoma: data from the Italian cohort of the ipilimumab expanded access program. Cancer invest. 32(4), 144-149 (2014).

11. Johnson DB, Pectasides E, Feld E et al. Sequencing treatment in BRAFV600 mutant melanoma: anti-PD-1 before and after BRAF inhibition. J. Immunother. 40(1), 31-35 (2017).

-. This retrospective analysis of 114 patients with BRAF mutant metastatic melanoma who had received anti-PD-1/PD-L1 treatments either before or after a BRAF inhibitor (alone or with a MEK inhibition) demonstrated similar overall survival irrespective of sequence and a $41 \%$ response rate for $1 \mathrm{~L}$ anti-PD-1/PDL1 therapy. 
12. Donia M, Ellebaek E, Ollegaard TH et al. The real-world impact of modern treatments on the survival of patients with metastatic melanoma. Eur. J. Cancer 108, 25-32 (2018).

13. Polkowska M, Ekk-Cierniakowski P, Czepielewska E, Kozlowska-Wojciechowska M. Efficacy and safety of BRAF inhibitors and anti-CTLA4 antibody in melanoma patients-real-world data. Eur. J. Clin. Pharmacol. doi:10.1007/s00228-018-2590-6 (2018). (Epub ahead of print).

14. Sassolas B, Leccia MT, Godard C et al. Real-world treatment patterns and clinical outcomes in advanced cutaneous melanoma patients in France. J Eur. Acad. Dermatol. Venereol. 32(4), 587-594 (2018).

15. Eisenhauer EA, Therasse P, Bogaerts J et al. New response evaluation criteria in solid tumours: revised RECIST guideline (version 1.1). Eur. J. Cancer 45(2), 228-247 (2009).

16. Wong GY, Mason WM. The hierarchical logistic regression model for multilevel analysis. J. Am. Stat. Assoc. 80(391), 513-524 (1985).

17. Long GV, Flaherty KT, Stroyakovskiy D et al. Dabrafenib plus trametinib versus dabrafenib monotherapy in patients with metastatic BRAF V600E/K-mutant melanoma: long-term survival and safety analysis of a Phase III study. Ann. Oncol. 28(7), 1631-1639 (2017).

18. Wolchok JD, Chiarion-Sileni V, Gonzalez R et al. Overall survival with combined nivolumab and ipilimumab in advanced melanoma. N. Engl. J. Med. 377(14), 1345-1356 (2017).

19. Daud A, Gill J, Kamra S, Chen L, Ahuja A. Indirect treatment comparison of dabrafenib plus trametinib versus vemurafenib plus cobimetinib in previously untreated metastatic melanoma patients. J. Hematol. Oncol. 10(1), 3 (2017).

20. Carlino MS, Long GV, Schadendorf D et al. Outcomes by line of therapy and programmed death ligand 1 expression in patients with advanced melanoma treated with pembrolizumab or ipilimumab in KEYNOTE-006: a randomised clinical trial. Eur. J. Cancer 101, 236-243 (2018).

21. Schachter J, Ribas A, Long GV et al. Pembrolizumab versus ipilimumab for advanced melanoma: final overall survival results of a multicentre, randomised, open-label Phase III study (KEYNOTE-006). Lancet 390(10105), 1853-1862 (2017).

22. Pires da Silva I, Wang KYX, Wilmott JS et al. Distinct molecular profiles and immunotherapy treatment outcomes of V600E and V600K BRAF-mutant melanoma. Clin. Cancer Res. (2019).

23. Robert C, Karaszewska B, Schachter J et al. Improved overall survival in melanoma with combined dabrafenib and trametinib. N. Engl. J. Med. 372(1), 30-39 (2015). 